\title{
Automatic object extraction from VHR satellite SAR images using Pulse Coupled Neural Networks
}

\author{
Fabio Del Frate**a ${ }^{\mathrm{a}}$, Daniele Latini ${ }^{\mathrm{a}}$, Chiara Pratola ${ }^{\mathrm{a}}$

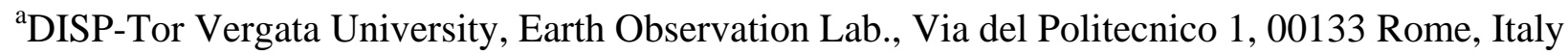

\begin{abstract}
An unsupervised neural network approach for automatically extracting objects of interest from very high resolution (VHR) SAR images is proposed. The methodology relies on the use of Pulse-Coupled Neural Networks (PCNN), an interesting novel technique based on models of the visual cortex of small mammals. Different applications are considered. In a first case the extraction procedure is focused on the detection of buildings. In a second case the extraction of the perimeter of a dark spot representing an oil spill in a SAR image is considered. The performance given by the PCNN is evaluated and critically discussed for a set of new generation of X-band SAR images taken by COSMOSkymed and TerraSAR-X systems.
\end{abstract}

Keywords: SAR, image segmentation, PCNN, oil spill detection, building extraction, COSMO-Skymed, TerraSAR-X 